\title{
Common fixed point theorems via implicit relations
}

\author{
Abdelkrim Aliouche
}




\title{
COMMON FIXED POINT THEOREMS VIA IMPLICIT RELATIONS
}

\author{
ABDELKRIM ALIOUCHE
}

Received 3 December, 2007

\begin{abstract}
We prove common fixed point theorems for four mappings satisfying implicit relations without decreasing assumption which improve theorems of $[1,13,14]$. We also prove common fixed point theorems for four mappings satisfying implicit relations which generalize theorems of $[3,4]$.
\end{abstract}

2000 Mathematics Subject Classification: 54H25, 47H10

Keywords: weakly compatible, common fixed point, metric space

\section{INTRODUCTION}

Let $S$ and $T$ be self-mappings of a metric space $(X, d) . S$ and $T$ are commuting if $S T x=T S x$ for all $x \in X$. Sessa [15] defined $S$ and $T$ to be weakly commuting if, for all $x \in X$,

$$
d(S T x, T S x) \leq d(T x, S x) .
$$

Jungck [7] defined $S$ and $T$ to be compatible, as a generalization of the weakly commuting property, if

$$
\lim _{n \rightarrow \infty} d\left(S T x_{n}, T S x_{n}\right)=0
$$

whenever $\left\{x_{n}\right\}$ is a sequence in $X$ such that $\lim _{n \rightarrow \infty} S x_{n}=\lim _{n \rightarrow \infty} T x_{n}=t$ for some $t \in X$.

It is easy to show that "commuting" implies "weakly commuting," which, in turn, implies "compatible," and there are examples in the literature justifying that the inclusions are proper (see $[7,15])$.

Jungck et al. [6] defined $S$ and $T$ to be compatible mappings of type (A) if

$$
\lim _{n \rightarrow \infty} d\left(S T x_{n}, T^{2} x_{n}\right)=0 \quad \text { and } \quad \lim _{n \rightarrow \infty} d\left(T S x_{n}, S^{2} x_{n}\right)=0
$$

whenever $\left\{x_{n}\right\}$ is a sequence in $X$ such that $\lim _{n \rightarrow \infty} S x_{n}=\lim _{n \rightarrow \infty} T x_{n}=t$ for some $t \in X$.

Clearly, "weakly commuting" implies "compatible of type (A)". By [6], the converse is not true, and the two concepts of compatibility are independent. 
Recently, Pathak and Khan [12] defined $S$ and $T$ to be compatible mappings of type $(B)$, as a generalization of compatible mappings of type (A), if

$$
\begin{aligned}
& \lim _{n \rightarrow \infty} d\left(T S x_{n}, S^{2} x_{n}\right) \leq \frac{1}{2}\left[\lim _{n \rightarrow \infty} d\left(T S x_{n}, T t\right)+\lim _{n \rightarrow \infty} d\left(T t, T^{2} x_{n}\right)\right], \\
& \lim _{n \rightarrow \infty} d\left(S T x_{n}, T^{2} x_{n}\right) \leq \frac{1}{2}\left[\lim _{n \rightarrow \infty} d\left(S T x_{n}, S t\right)+\lim _{n \rightarrow \infty} d\left(S t, S^{2} x_{n}\right)\right]
\end{aligned}
$$

whenever $\left\{x_{n}\right\}$ is a sequence in $X$ such that $\lim _{n \rightarrow \infty} S x_{n}=\lim _{n \rightarrow \infty} T x_{n}=t$ for some $t \in X$.

Clearly, compatible mappings of type (A) are compatible mappings of type (B), but the converse is not true [9]. However, the notions of compatibility, compatibility of type (A), and compatibility of type (B) are equivalent to one another if $S$ and $T$ are continuous [12].

Pathak et al. [10] defined $S$ and $T$ to be compatible mappings of type $(P)$ if

$$
\lim _{n \rightarrow \infty} d\left(S^{2} x_{n}, T^{2} x_{n}\right)=0
$$

whenever $\left\{x_{n}\right\}$ is a sequence in $X$ such that $\lim _{n \rightarrow \infty} S x_{n}=\lim _{n \rightarrow \infty} T x_{n}=t$ for some $t \in X$.

The notions of compatibility, compatibility of type (A), and compatibility of type (P) are mutually equivalent if $S$ and $T$ are continuous [10].

Pathak et al. [11] defined $S$ and $T$ to be compatible mappings of type $(C)$ as a generalization of compatible mappings of type (A) if

$$
\begin{aligned}
\lim _{n \rightarrow \infty} d\left(T S x_{n}, S^{2} x_{n}\right) \leq & \frac{1}{3}\left(\lim _{n \rightarrow \infty} d\left(T S x_{n}, T t\right)+\lim _{n \rightarrow \infty} d\left(T t, S^{2} x_{n}\right)\right. \\
& \left.+\lim _{n \rightarrow \infty} d\left(T t, T^{2} x_{n}\right)\right), \\
\lim _{n \rightarrow \infty} d\left(S T x_{n}, T^{2} x_{n}\right) \leq & \frac{1}{3}\left(\lim _{n \rightarrow \infty} d\left(S T x_{n}, S t\right)+\lim _{n \rightarrow \infty} d\left(S t, T^{2} x_{n}\right)\right. \\
& \left.+\lim _{n \rightarrow \infty} d\left(S t, S^{2} x_{n}\right)\right)
\end{aligned}
$$

whenever $\left\{x_{n}\right\}$ is a sequence in $X$ such that $\lim _{n \rightarrow \infty} S x_{n}=\lim _{n \rightarrow \infty} T x_{n}=t$ for some $t \in X$.

Clearly, compatible mappings of type (A) are also compatible mappings of type (C), but the converse implication is not true [11]. However, the properties of compatibility of type (A) and compatibility of type (C) are mutually equivalent if $S$ and $T$ are continuous (see [11]).

\section{Preliminaries}

Definition 1 ([5]). $S$ and $T$ are said to be weakly compatible if they commute at their coincidence points, i. e., the equality $S u=T u$ for some $u \in X$ implies that $S T u=T S u$. 
Lemma 1 ([6, 7, 10-12]). If $S$ and $T$ are compatible, or compatible of type (A), or compatible of type $(\mathrm{P})$, or compatible of type $(\mathrm{B})$, or compatible of type $(\mathrm{C})$, then they are weakly compatible.

The converse is not true in general, see [2].

Definition 2 ([8]). $S$ and $T$ are said to be $R$-weakly commuting at a point $x \in X$ if for some $R>0$

$$
d(S T x, T S x) \leq R d(T x, S x) .
$$

Definition 3 ([9]). $S$ and $T$ are said to be pointwise R-weakly commuting on $X$ if, given an $x \in X$, there exists an $R>0$ such that (2.1) holds.

It was proved in [9] that the $R$-weak commutativity is equivalent to commutativity at coincidence points, i. e., $S$ and $T$ are pointwise $R$-weakly commuting if and only if they are weakly compatible.

Let $\mathbb{R}_{+}$be the set of all non-negative reals numbers and $K_{6}$ the family of all continuous mappings $K\left(t_{1}, t_{2}, t_{3}, t_{4}, t_{5}, t_{6}\right): \mathbb{R}_{+}^{6} \rightarrow \mathbb{R}$ with $t_{3}+t_{4} \neq 0$ satisfying the following conditions:

$\left(K_{1}\right) K$ is decreasing in variables $t_{5}$ and $t_{6}$.

$\left(K_{2}\right)$ there exists $0 \leq h<1$ such that for all $u, v \geq 0$ with

$\left(K_{a}\right) K(u, v, v, u, u+v, 0) \leq 0$ or

$\left(K_{b}\right) K(u, v, u, v, 0, u+v) \leq 0$

we have $u \leq h v$.

The following theorem was proved in [13].

Theorem 1. Let $S, T, I$ and $J$ be self-mappings of a metric space $(X, d)$ satisfying

(a) $S(X) \subset J(X)$ and $T(X) \subset I(X)$,

(b) the inequality

$$
\begin{aligned}
& F(d(S x, T y), d(I x, J y), d(I x, S x), d(J y, T y), d(I x, T y), d(S x, J y)) \leq 0 \\
& \text { holds for all } x, y \in X \text { and } F \in K_{6} \text { satisfies conditions }\left(K_{1}\right) \text { and }\left(K_{2}\right) \text { if } \\
& d(I x, S x)+d(J y, T y) \neq 0, \text { or } \\
& d(S x, T y)=0 \text { if }(I x, S x)+d(J y, T y)=0,
\end{aligned}
$$

(c) the pairs $(S, I)$ and $(T, J)$ are weakly compatible.

If one of $S(X), T(X), I(X)$ and $J(X)$ is a complete subspace of $X$, then, $S, T, I$ and $J$ have a unique common fixed point $z$ in $X$. Further, $z$ is the unique common fixed point of $S$ and $I$ and $T$ and $J$.

It is our purpose in this paper to prove common fixed point theorems for weakly compatible mappings satisfying implicit relations without condition $\left(K_{1}\right)$, which improve results of Ali and Imdad [1] and Popa [13,14]. We also prove a common fixed 
point theorem for weakly compatible mappings satisfying implicit relations which generalizes results of Jeong and Rhoades [4] and Jeong [3].

\section{IMPLICIT RELATIONS}

Let $F_{6}$ be the family of all continuous mappings $F\left(t_{1}, t_{2}, t_{3}, t_{4}, t_{5}, t_{6}\right): \mathbb{R}_{+}^{6} \rightarrow \mathbb{R}$ with $t_{3}+t_{4} \neq 0$ satisfying the following condition:

$\left(F_{1}\right)$ there exists $0 \leq h<1$ such that for all $u, v, w \geq 0$ with

$\left(F_{a}\right) F(u, v, v, u, w, 0) \leq 0$ or

$\left(F_{b}\right) \quad F(u, v, u, v, 0, w) \leq 0$

we have $u \leq h v$.

Example 1. $F\left(t_{1}, t_{2}, t_{3}, t_{4}, t_{5}, t_{6}\right)=t_{1}-a \frac{t_{2} t_{3}}{t_{3}+t_{4}}-b \frac{t_{4} t_{5}}{t_{5}+t_{6}+1}$, where $0<a, b<1$ and $a+b<1$.

$\left(F_{1}\right)$ : Let $u, v, w \geq 0$ and $F(u, v, v, u, w, 0)=u-a \frac{v^{2}}{u+v}-b \frac{u w}{w+1} \leq 0$. Then, $u \leq h_{1} v$, where $h_{1}=\frac{a}{1-b}<1$. Similarly, if $F(u, v, u, v, 0, w) \leq 0$ then $u \leq h_{2} v$, where $h_{2}=a<1$. We take $h=\max \left\{h_{1}, h_{2}\right\}<1$.

Example 2. $F\left(t_{1}, t_{2}, t_{3}, t_{4}, t_{5}, t_{6}\right)=t_{1}-a \frac{t_{2} t_{4}}{t_{3}+t_{4}}-b \frac{t_{3} t_{6}}{t_{5}+t_{6}+1}$, where $0<a, b<1$ and $a+b<1$. The condition $\left(F_{1}\right)$ can be verified as in Example 1 .

Let $H_{6}$ be the family of all continuous mappings $H\left(t_{1}, t_{2}, t_{3}, t_{4}, t_{5}, t_{6}\right): \mathbb{R}_{+}^{6} \rightarrow \mathbb{R}$ with $t_{5}+t_{6} \neq 0$ satisfying the following conditions:

$\left(H_{1}\right)$ there exists $0 \leq h<1$ such that for all $u, v, w \geq 0$ with

$\left(H_{a}\right) H(u, v, v, u, w, 0) \leq 0$ or

$\left(H_{b}\right) H(u, v, u, v, 0, w) \leq 0$

we have $u \leq h v$.

$\left(H_{2}\right) H(u, u, 0,0, u, u)>0$ for all $u>0$.

Example 3. $H\left(t_{1}, t_{2}, t_{3}, t_{4}, t_{5}, t_{6}\right)=t_{1}-a \frac{t_{3} t_{6}+t_{4} t_{5}}{t_{5}+t_{6}}-b t_{2}$, where $a, b>0$ and $a+$ $b<1$.

$\left(H_{1}\right)$ : Let $u, v, w \geq 0$ and $H(u, v, v, u, w, 0)=u-a u-b v \leq 0$. Then, $u \leq h v$, where $h=\frac{b}{1-a}<1$. Similarly, if $H(u, v, u, v, 0, w) \leq 0$ then $u \leq h v$.

$\left(H_{2}\right): H(u, u, 0,0, u, u)=(1-b) u>0$ for all $u>0$.

Example 4. $H\left(t_{1}, t_{2}, t_{3}, t_{4}, t_{5}, t_{6}\right)=t_{1}-\frac{a t_{3} t_{5}+b t_{4} t_{6}}{t_{5}+t_{6}}-c t_{2}$, where $a, b, c>0$ and $a+b+c<1$.

$\left(H_{1}\right)$ : Let $u, v, w \geq 0$ and $H(u, v, v, u, w, 0)=u-a v-c v \leq 0$. Then, $u \leq h_{1} v$, where $h_{1}=a+c<1$. Similarly, if $H(u, v, u, v, 0, w) \leq 0$ then $u \leq h_{2} v$, where $h_{2}=b+c<1$. We take $h=\max \left\{h_{1}, h_{2}\right\}<1$.

$\left(H_{2}\right): H(u, u, 0,0, u, u)=(1-c) u>0$ for all $u>0$.

Let $G_{6}$ be the family of all continuous mappings $G\left(t_{1}, t_{2}, t_{3}, t_{4}, t_{5}, t_{6}\right): \mathbb{R}_{+}^{6} \rightarrow \mathbb{R}$ with $t_{2}+t_{4} \neq 0$ satisfying the following conditions: 
$\left(G_{1}\right) G$ is decreasing in variables $t_{5}$ and $t_{6}$.

$\left(G_{2}\right)$ there exists $0 \leq h<1$ such that for all $u, v \geq 0$ with

$\left(G_{a}\right) G(u, v, v, u, u+v, 0) \leq 0$ or

$\left(G_{b}\right) G(u, v, u, v, 0, u+v) \leq 0$

we have $u \leq h v$.

$\left(G_{3}\right) G(u, u, 0,0, u, u)>0$ for all $u>0$.

Example 5. $G\left(t_{1}, t_{2}, t_{3}, t_{4}, t_{5}, t_{6}\right)=t_{1}-a_{1} \frac{t_{2} t_{5}}{t_{2}+t_{4}}-a_{2}\left(t_{3}+t_{4}\right)-a_{3}\left(t_{5}+t_{6}\right)-a_{4} t_{2}$, where $a_{1}, a_{2}, a_{3}, a_{4}>0$ and $a_{1}+2 a_{2}+2 a_{3}+a_{4}<1$.

$\left(G_{1}\right)$ : It is clear.

$\left(G_{2}\right)$ : Let $u, v \geq 0$ and $G(u, v, v, u, u+v, 0)=u-a_{1} \frac{v(u+v)}{u+v}-a_{2}(u+v)-$ $a_{3}(u+v)-a_{4} v \leq 0$. Then $u \leq h_{1} v$, where $h_{1}=\frac{a_{1}+a_{2}+a_{3}+a_{4}}{1-a_{2}-a_{3}}<1$. Similarly, if $G(u, v, u, v, 0, u+v) \leq 0$ then $u \leq h_{2} v$, where $h_{2}=\frac{a_{2}+a_{3}+a_{4}}{1-a_{2}-a_{3}}<1$. We take $h=\max \left\{h_{1}, h_{2}\right\}<1$.

$\left(G_{3}\right): G(u, u, 0,0, u, u)=\left(1-a_{1}-2 a_{3}-a_{4}\right) u>0$ for all $u>0$.

Let $C_{6}$ be the family of all continuous mappings $C\left(t_{1}, t_{2}, t_{3}, t_{4}, t_{5}, t_{6}\right): \mathbb{R}_{+}^{6} \rightarrow \mathbb{R}$ with $t_{2}+t_{4} \neq 0$ satisfying the following conditions:

$\left(C_{1}\right)$ there exists $0 \leq h<1$ such that for all $u, v, w \geq 0$ with

$\left(C_{a}\right) C(u, v, v, u, w, 0) \leq 0$ or

$\left(C_{b}\right) C(u, v, u, v, 0, w) \leq 0$

we have $u \leq h v$.

$\left(C_{2}\right) C(u, u, 0,0, u, u)>0$ for all $u>0$.

Example 6. $C\left(t_{1}, t_{2}, t_{3}, t_{4}, t_{5}, t_{6}\right)=t_{1}-a \frac{t_{2} t_{4}}{t_{2}+t_{4}}-b \frac{t_{3} t_{5}}{t_{5}+t_{6}+1}$, where $0<a, b<1$ and $a+b<1$.

$\left(C_{1}\right):$ Let $u, v, w \geq 0$ and $C(u, v, v, u, w, 0)=u-a \frac{u v}{u+v}-b \frac{v w}{w+1} \leq 0$. Then $u \leq h_{1} v$, where $h_{1}=a+b<1$. Similarly, if $C(u, v, u, v, 0, w) \leq 0$ then $u \leq h_{2} v$, $h_{2}=\frac{a}{2}<1$. We take $h=\max \left\{h_{1}, h_{2}\right\}<1$.

$\left(C_{2}\right): C(u, u, 0,0, u, u)=u>0$ for all $u>0$.

Example 7. $C\left(t_{1}, t_{2}, t_{3}, t_{4}, t_{5}, t_{6}\right)=t_{1}-a \frac{t_{2} t_{4}}{t_{2}+t_{4}}-b \frac{t_{3} t_{6}}{t_{5}+t_{6}+1}$, where $0<a, b<1$ and $a+2 b<2$. $\left(C_{1}\right)$ and $\left(C_{2}\right)$ follow as in Example 6.

\section{MAin RESUltS}

Theorem 2. Let $f, g, S$ and $T$ be self-mappings of a metric space $(X, d)$ satisfying the following conditions:

$$
\begin{gathered}
S(X) \subset g(X), \quad T(X) \subset f(X), \\
F(d(S x, T y), d(f x, g y), d(f x, S x), d(g y, T y), d(f x, T y), d(S x, g y)) \leq 0
\end{gathered}
$$


for all $x, y \in X$ and $F \in F_{6}$ satisfies $\left(F_{1}\right)$ if $d(f x, S x)+d(g y, T y) \neq 0$, or

$$
d(S x, T y)=0 \quad \text { if } \quad d(f x, S x)+d(g y, T y)=0 .
$$

Suppose that one of $S(X), T(X), f(X)$, and $g(X)$ is a complete subspace of $X$ and the pairs $(S, f)$ and $(T, g)$ are weakly compatible.

Then, $f, g, S$, and $T$ have a unique common fixed point $z$ in $X$. Further, $z$ is the unique common fixed point of $S$ and $f$ and $T$ and $g$.

Proof. Let $x_{0}$ be an arbitrary point in $X$. By (4.1), we can define inductively a sequence $\left\{y_{n}\right\}$ in $X$ such that

$$
y_{2 n}=S x_{2 n}=g x_{2 n+1}, y_{2 n+1}=f x_{2 n+2}=T x_{2 n+1} \quad \text { for } n=0,1,2, \ldots
$$

If

$$
d\left(f x_{2 n}, S x_{2 n}\right)+d\left(g x_{2 n+1}, T x_{2 n+1}\right) \neq 0,
$$

then, using (4.2) and (4.4), we have

$$
\begin{gathered}
F\left(d\left(S x_{2 n}, T x_{2 n+1}\right), d\left(f x_{2 n}, g x_{2 n+1}\right), d\left(f x_{2 n}, S x_{2 n}\right), d\left(g x_{2 n+1}, T x_{2 n+1}\right),\right. \\
\left.d\left(f x_{2 n}, T x_{2 n+1}\right), d\left(S x_{2 n}, g x_{2 n+1}\right)\right) \\
=F\left(d\left(y_{2 n}, y_{2 n+1}\right), d\left(y_{2 n-1}, y_{2 n}\right), d\left(y_{2 n-1}, y_{2 n}\right), d\left(y_{2 n}, y_{2 n+1}\right),\right. \\
\left.d\left(y_{2 n-1}, y_{2 n+1}\right), 0\right) \leq 0
\end{gathered}
$$

By $\left(F_{a}\right)$, we get

$$
d\left(y_{2 n}, y_{2 n+1}\right) \leq h d\left(y_{2 n-1}, y_{2 n}\right) .
$$

Similarly, if

$$
d\left(f x_{2 n+2}, S x_{2 n+2}\right)+d\left(g x_{2 n+1}, T x_{2 n+1}\right) \neq 0 .
$$

we obtain

$$
d\left(y_{2 n+1}, y_{2 n+2}\right) \leq h d\left(y_{2 n}, y_{2 n+1}\right) .
$$

Therefore,

$$
d\left(y_{n}, y_{n+1}\right) \leq h d\left(y_{n-1}, y_{n}\right) .
$$

Thus, $\left\{y_{n}\right\}$ is a Cauchy sequence in $X$ and, therefore, the subsequence $\left\{y_{2 n}\right\}=$ $\left\{g x_{2 n+1}\right\} \subset g(X)$ is a Cauchy sequence in $g(X)$. Since $g(X)$ is complete, it converges to a point $z=g v$ for some $v \in X$.

Therefore, the sequence $\left\{y_{n}\right\}$ converges also to $z$ and the subsequences $\left\{S x_{2 n}\right\}$, $\left\{T x_{2 n+1}\right\}$, and $\left\{f x_{2 n+2}\right\}$ converge to $z$.

If $z \neq T v$, using (4.2), we obtain

$$
\begin{aligned}
& F\left(\left(d\left(S x_{2 n}, T v\right), d\left(f x_{2 n}, g v\right), d\left(f x_{2 n}, S x_{2 n}\right), d(g v, T v), d\left(f x_{2 n}, T v\right),\right.\right. \\
& \left.\quad d\left(S x_{2 n}, g v\right)\right) \leq 0
\end{aligned}
$$

Letting $n \rightarrow \infty$ and using the continuity of $F$, we obtain

$$
F(d(z, T v), 0,0, d(z, T v), d(z, T v), 0) \leq 0 .
$$


By $\left(F_{a}\right)$, we get $z=T v=g v$. Since $T(X) \subset f(X)$, there exists an $u \in X$ such that $z=f u=T v$.

If $z \neq S u$, using (4.2) we have

$$
\begin{array}{r}
F(d(S u, T v), d(f u, g v), d(f u, S u), d(g v, T v), d(f u, T v), d(S u, g v)) \\
=F(d(S u, z), 0, d(z, S u), 0,0, d(S u, z)) \leq 0 .
\end{array}
$$

By $\left(F_{b}\right)$, we get $z=S u=f u$. Since the pairs $(S, f)$ and $(T, g)$ are weakly compatible, we get $f z=S z$ and $g z=T z$. Since $d(f z, S z)+d(g v, T v)=0$, in view of (4.3), it follows that $d(S z, T v)=0$, i. e., $z=S z=f z$.

Similarly, we can prove that $z=g z=T z$. Hence, $z$ is a common fixed point of $f, g, S$, and $T$.

The proof is similar if we suppose that, instead of $g(X)$, one of $S(X), T(X)$, and $f(X)$ is complete. The uniqueness of $z$ follows from (4.3).

Remark 1. As the function $F$ in Theorem 2 is not decreasing in variables $t_{5}$ and $t_{6}$, theorems of $[1,13,14]$ are not applicable in this case.

Considering Examples 1 and 2, we obtain two corollaries of Theorem 2.

Theorem 3. Let $f, g, S$, and $T$ be self-mappings of a metric space $(X, d)$ satisfying (4.1),

$$
H(d(S x, T y), d(f x, g y), d(f x, S x), d(g y, T y), d(f x, T y), d(S x, g y)) \leq 0
$$

for all $x, y \in X$ and $H \in H_{6}$ satisfies $\left(H_{1}\right)$ and $\left(H_{2}\right)$ if $d(f x, T y)+d(S x, g y) \neq 0$, or

$$
d(S x, T y)=0 \quad \text { if } \quad d(f x, T y)+d(S x, g y)=0 .
$$

Suppose that one of $S(X), T(X), f(X)$, and $g(X)$ is a complete subspace of $X$ and the pairs $(S, f)$ and $(T, g)$ are weakly compatible.

Then $f, g, S$, and $T$ have a unique common fixed point $z$ in $X$. Further, $z$ is the unique common fixed point of $S$ and $f$ and $T$ and $g$.

Proof. Let $x_{0}$ be an arbitrary point in $X$. By (4.1), we can define inductively a sequence $\left\{y_{n}\right\}$ in $X$ by (4.4).

If

or

$$
d\left(f x_{2 n}, T x_{2 n+1}\right)+d\left(S x_{2 n}, g x_{2 n+1}\right)=0
$$

$$
d\left(f x_{2 n+2}, T x_{2 n+1}\right)+d\left(g x_{2 n+1}, S x_{2 n+2}\right)=0
$$

for some $n$, the proof is similar to [14].

If

and

$$
d\left(f x_{2 n}, T x_{2 n+1}\right)+d\left(S x_{2 n}, g x_{2 n+1}\right) \neq 0
$$

$$
d\left(f x_{2 n+2}, T x_{2 n+1}\right)+d\left(g x_{2 n+1}, S x_{2 n+2}\right) \neq 0,
$$


as in the proof of Theorem 2, we obtain $z=f u=S u=g v=T v$.

Since the pairs $(S, f)$ and $(T, g)$ are weakly compatible, we get $f z=S z$ and $g z=T z$.

If $z \neq S z$, using (4.2) we have

$$
\begin{aligned}
& H(d(S z, T v), d(f z, g v), d(f z, S z), d(g v, T v), d(f z, T v), d(S z, g v)) \\
&=H(d(S z, z), d(S z, z), 0,0, d(S z, z), d(S z, z)) \leq 0 .
\end{aligned}
$$

which is a contradiction to $\left(H_{2}\right)$. Therefore, $z=S z=f z$.

Similarly, we can prove that $z=T z=g z$. Hence, $z$ is a common fixed point of $f, g, S$, and $T$. The uniqueness of $z$ follows from (4.5) and $\left(\mathrm{H}_{2}\right)$.

Theorem 3 generalizes [4, Theorem 5] and [3, Theorem 2].

Theorem 4. Let $f, g, S$, and $T$ be self-mappings of a metric space $(X, d)$ satisfying (4.1),

$$
G(d(S x, T y), d(f x, g y), d(f x, S x), d(g y, T y), d(f x, T y), d(S x, g y)) \leq 0
$$

for all $x, y \in X$ and $G \in G_{6}$ satisfies conditions $\left(G_{1}\right),\left(G_{2}\right)$, and $\left(G_{3}\right)$ if $d(f x, g y)+$ $d(g y, T y) \neq 0$, or

$$
d(S x, T y)=0 \quad \text { if } \quad d(f x, g y)+d(g y, T y)=0 .
$$

Suppose that one of $S(X), T(X), f(X)$, and $g(X)$ is a complete subspace of $X$ and the pairs $(S, f)$ and $(T, g)$ are weakly compatible.

Then, $f, g, S$, and $T$ have a unique common fixed point $z$ in $X$. Further, $z$ is the unique common fixed point of $S$ and $f$ and $T$ and $g$.

Proof. Let $x_{0}$ be an arbitrary point in $X$. By (4.1), we can define inductively a sequence $\left\{y_{n}\right\}$ in $X$ by (4.4).

If

$$
d\left(f x_{2 n}, T x_{2 n+1}\right)+d\left(S x_{2 n}, g x_{2 n+1}\right) \neq 0
$$

using (4.2) and (4.4) we have

$$
\begin{aligned}
& G\left(d\left(S x_{2 n}, T x_{2 n+1}\right), d\left(f x_{2 n}, g x_{2 n+1}\right), d\left(f x_{2 n}, S x_{2 n}\right), d\left(g x_{2 n+1}, T x_{2 n+1}\right),\right. \\
& \left.\quad d\left(f x_{2 n}, T x_{2 n+1}\right), d\left(S x_{2 n}, g x_{2 n+1}\right)\right) \\
& =G\left(d\left(y_{2 n}, y_{2 n+1}\right), d\left(y_{2 n-1}, y_{2 n}\right), d\left(y_{2 n-1}, y_{2 n}\right), d\left(y_{2 n}, y_{2 n+1}\right),\right. \\
& \left.\quad d\left(y_{2 n-1}, y_{2 n+1}\right), 0\right) \leq 0 .
\end{aligned}
$$

By $\left(G_{1}\right)$ we have

$$
\begin{aligned}
& G\left(d\left(y_{2 n}, y_{2 n+1}\right), d\left(y_{2 n-1}, y_{2 n}\right), d\left(y_{2 n-1}, y_{2 n}\right),\right. \\
& \left.\quad d\left(y_{2 n}, y_{2 n+1}\right), d\left(y_{2 n-1}, y_{2 n}\right)+d\left(y_{2 n}, y_{2 n+1}\right), 0\right) \leq 0 .
\end{aligned}
$$


By virtue of $\left(G_{a}\right)$, we get

$$
d\left(y_{2 n}, y_{2 n+1}\right) \leq h d\left(y_{2 n-1}, y_{2 n}\right) .
$$

In the same manner, if

$$
d\left(f x_{2 n+2}, S x_{2 n+2}\right)+d\left(g x_{2 n+1}, T x_{2 n+1}\right) \neq 0
$$

we obtain

$$
d\left(y_{2 n+1}, y_{2 n+2}\right) \leq h d\left(y_{2 n}, y_{2 n+1}\right) .
$$

Therefore,

$$
d\left(y_{n}, y_{n+1}\right) \leq h d\left(y_{n-1}, y_{n}\right) .
$$

As in the proofs of Theorems 2 and $3, z$ is a common fixed point of $f, g, S$, and $T$. The uniqueness of $z$ follows from (4.6) and $\left(G_{3}\right)$.

Remark 2. Theorem 4 generalizes [4, Theorem 9] and [3, Theorem 5].

Similarly to Theorem 3, we can prove the following statement which improves Theorem 4.

Theorem 5. Let $f, g, S$, and $T$ be self-mappings of a metric space $(X, d)$ satisfying (4.1),

$$
C(d(S x, T y), d(f x, g y), d(f x, S x), d(g y, T y), d(f x, T y), d(S x, g y)) \leq 0
$$

for all $x, y \in X$ and $C \in C_{6}$ satisfies $\left(C_{1}\right)$ and $\left(C_{2}\right)$ if $d(f x, g y)+d(g y, T y) \neq 0$, or

$$
d(S x, T y)=0 \quad \text { if } \quad d(f x, g y)+d(g y, T y)=0 .
$$

Suppose that one of $S(X) T(X), f(X)$, and $g(X)$ is a complete subspace of $X$ and the pairs $(S, f)$ and $(T, g)$ are weakly compatible.

Then, $f, g, S$, and $T$ have a unique common fixed point $z$ in $X$. Further, $z$ is the unique common fixed point of $S$ and $f$ and $T$ and $g$.

If $S=T$ or $f=g$ or $S=T$ and $f=g$, or $f=g=I$, where $I$ denotes the identity mapping, or $S=T$ and $f=g=I$ in Theorems $2,3,4$, and 5, one can obtain several corollaries.

\section{REFERENCES}

[1] J. Ali and M. Imdad, "Unifying a multitude of common fixed point theorems employing an implicit relation," Commun. Korean Math. Soc., vol. 24, no. 1, pp. 41-55, 2009. [Online]. Available: http://dx.doi.org/10.4134/CKMS.2009.24.1.041

[2] A. Aliouche, "A common fixed point theorem for weakly compatible mappings in compact metric spaces satisfying an implicit relation," Sarajevo J. Math., vol. 3(15), no. 1, pp. 123-130, 2007.

[3] G. S. Jeong, "Some fixed point theorems under weak conditions," JP J. Geom. Topol., vol. 5, no. 1, pp. 83-96, 2005.

[4] G. S. Jeong and B. E. Rhoades, "Some remarks for improving fixed point theorems for more than two maps,” Indian J. Pure Appl. Math., vol. 28, no. 9, pp. 1177-1196, 1997. 
[5] G. Jungck, "Common fixed points for noncontinuous nonself maps on nonmetric spaces," Far East J. Math. Sci., vol. 4, no. 2, pp. 199-215, 1996.

[6] G. Jungck, P. P. Murthy, and Y. J. Cho, "Compatible mappings of type $(A)$ and common fixed points," Math. Japon., vol. 38, no. 2, pp. 381-390, 1993

[7] G. Jungck, "Compatible mappings and common fixed points," Internat. J. Math. Math. Sci., vol. 9, no. 4, pp. 771-779, 1986. [Online]. Available: http://dx.doi.org/10.1155/S0161171286000935

[8] R. P. Pant, "Common fixed points of noncommuting mappings," J. Math. Anal. Appl., vol. 188, no. 2, pp. 436-440, 1994. [Online]. Available: http://dx.doi.org/10.1006/jmaa.1994.1437

[9] R. P. Pant, "Common fixed points of four mappings," Bull. Calcutta Math. Soc., vol. 90, no. 4, pp. 281-286, 1998.

[10] H. K. Pathak, Y. J. Cho, S. M. Kang, and B. S. Lee, "Fixed point theorems for compatible mappings of type (P) and applications to dynamic programming," Matematiche (Catania), vol. 50, no. 1, pp. 15-33, 1995.

[11] H. K. Pathak, Y. J. Cho, S. M. Kang, and B. Madharia, "Compatible mappings of type $(C)$ and common fixed point theorems of Greguš type," Demonstratio Math., vol. 31, no. 3, pp. 499-518, 1998.

[12] H. K. Pathak and M. S. Khan, "Compatible mappings of type (B) and common fixed point theorems of Greguš type," Czechoslovak Math. J., vol. 45(120), no. 4, pp. 685-698, 1995.

[13] V. Popa, "Some fixed point theorems for weakly compatible mappings," Rad. Mat., vol. 10, no. 2, pp. 245-252, 2001.

[14] V. Popa, "A general fixed point theorem for four weakly compatible mappings satisfying an implicit relation," Filomat, no. 19, pp. 45-51, 2005.

[15] S. Sessa, "On a weak commutativity condition of mappings in fixed point considerations," Publ. Inst. Math. (Beograd) (N.S.), vol. 32(46), pp. 149-153, 1982.

Author's address

Abdelkrim Aliouche

Department of Mathematics, University of Larbi Ben M'Hidi, Oum-El-Bouaghi, 04000, Algeria

E-mail address: alioumath@yahoo.fr 